\title{
Determination of Grain Boundary Volume Expansion by HREM*
}

\author{
M. I. Buckett and Karl L. Merkle \\ Materials Science Division \\ Argonne National Laboratory, Argonne, IL 60439
}

\section{March 1994}

The submitted manuscript has been authored
by contractor of the U.S. Government
under contract No. W-31-109-ENG-38.
Accordingly, the U.S. Covernment retalns a
nonexclusive, royalty-tree llcense to publish
or reproduce the published form of this
contrbution, or allow others to do so, for
U.S. Government purposes.

\section{DISCLAIMER}

\begin{abstract}
This report was prepared as an account of work sponsored by an agency of the United States Government. Neither the United States Government nor any agency thereof, nor any of their employees, makes any warranty, express or implied, or assumes any legal liability or responsibility for the accuracy, completeness, or usefulness of any information, apparatus, product, or process disclosed, or represents that its use would not infringe privately owned rights. Reference herein to any specific commercial product, process, or service by trade name, trademark, manufacturer, or otherwise does not necessarily constitute or imply its endorsement, recommendation, or favoring by the United States Government or any agency thereof. The views and opinions of authors expressed herein do not necessarily state or reflect those of the United States Government or any agency thereof.
\end{abstract}

INVITED PAPER presented at the Symposium on High-Voltage and HighResolution Electron Microscopy, Stuttgart, Germany, February 21-24, 1994; to be published in Ultramicroscopy.

\footnotetext{
*Work supported by the U.S. Department of Energy, Basic Energy Sciences-Materials Science,
} under contract \#W-31-109-ENG-38. 


\title{
Determination of Grain Boundary Volume Expansion by HREM
}

M. I. Buckett and Karl L. Merkle, Argonne National Laboratory, Argonne, IL 60439

\begin{abstract}
High resolution electron microscopy (HREM) has provided invaluable insight into the structure of grain boundaries at the atomistic level. Quantitative HREM methods are now in development which, combined with atomistic simulations, can provide further insight into grain boundary structure-energy correlations. For example, the volume expansion (or excess free volume, $\delta$ ), a thermodynamic parameter directly related to the grain boundary energy, is represented by the normal component of the rigid body translation. Although its magnitude is small, it can be determined experimentally using statistical techniques which locate and fit the peak and valley positions in an experimental HREM image - i.e., a direct measurement of the lattice fringe displacements is made. We have modified the lattice fringe displacement technique such that a measurement accuracy of better than $\pm 0.002 \mathrm{a}_{o}$ can be achieved. Precise knowledge of the position and intensity of the image contrast and a detailed understanding of the sources of error are required. This paper provides a detailed description of the lattice fringe displacement technique as well as an analysis of the sources of error in the measurement.
\end{abstract}

\section{INTRODUCTION AND BACKGROUND}

It is well-established in the grain boundary literature that the rigid body translation provides a major relaxation mechanism for the minimization of grain boundary energy in a wide range of boundary types [1-3]. The component of the rigid body translation normal to the boundary represents the volume expansion or excess free volume of the particular boundary. This quantity is defined as the free volume change per unit area of interface (at constant $T, P, N$ ) and is expressed in units of length. In principle, it is one of the purely thermodynamic parameters which can be directly measured. What is required, however, is a quantitative method sensitive to the small displacements (of the order of $1 / 10$ to $1 / 100$ of the lattice parameter) which represent the volume expansion.

A number of quantitative electron microscopy techniques have been developed to characterize grain boundary rigid body displacements. These include the $\alpha$-fringe [4,5], Moire fringe [6] and Fresnel fringe [7-9] methods, each of which uses the relative displacement of their respective interference fringes across a boundary to evaluate parameters such as volume expansion, and the lattice fringe method [10-12] which directly evaluates the volume expansion from an HREM image. Of the four methods, it can be argued that the lattice fringe displacement technique is the most direct in that it gives an absolute rather than a relative measurement. 
Efforts to relate the experimental volume expansion measurements to theoretical results have thus far met with only limited success. For example, shown in Table I is a sampling of experimental and theoretical data which have determined grain boundary volume expansion in $\Sigma 3$ twin boundaries. While the lattice statics computer simulation studies suggest close to a linear relation between the grain boundary volume expansion and grain boundary energy [13], the experimental results generally show considerable deviation from the theoretical calculations. However, the data from the simulations depend on the type of potential used. In order for the experiments to be valuable as a test of the theoretical calculations, it is necessary to improve the accuracy of the measurements to substantially better than the spread of values from the theoretical results using different potentials. The lattice fringe displacement method is potentially the n:ost direct method to accomplish this, thus it was chosen in the present study for evaluation in terms of measurement and acquisition errors. What follows is first a description of the technique as it has been modified by the present authors. Secondly, the statistical errors involved in making the measurement are investigated. Finally, the potential errors involved in the actual image acquisition process will be examined.

\section{THE LATTICE FRINGE DISPLACEMENT METHOD}

The lattice fringe displacement method was originally proposed by Stobbs and coworkers $[10,11]$ and modified by Merkle [12] to be applicable for the more general case of asymmetric as well as symmetric boundaries. It is based on the premise that the relative position of the lattice fringes (which make up the HREM structure image) on opposite sides of a boundary can be determined with a high degree of precision. Although the appearance of a HREM structure image is known to be affected by a number of factors - dependent on both the specimen and the microscope parameters - the relative position of the lattice fringe should accurately reflect the relative shift of the projected potentials when the imaging conditions are suitable. The lattice fringe positions on opposite sides of a boundary can thus be measured and the relative shift evaluated. We have modified the technique to further minimize the operator error associated with collecting the data and have found that measurements to better than $\pm 0.002 \mathrm{a}_{0}$ (normalized to the lattice parameter, $\mathrm{a}_{0}$ ) may be obtained under ideal conditions.

The technique first assumes that an HREM image of a tilt grain boundary taken under axial illumination conditions is available in digitized form. Shown in Figure 1 is a schematic diagram illustrating the typical experimental set-up developed by the authors. It involves first framing a set of lattice peaks (planes) on opposite sides of the boundary to use as a data set, where W marks the boundary width in the particular data set, $d$ represents the interplanar distance, and s marks the projected interatomic distance along the row of selected peaks. Within the framed areas on each side of the boundary, a two-dimensional peak search is performed which loops through every pixel. At each pixel a test is performed to see if it is the position of the highest intensity within a user-specified 
range. An iterative routine is then performed which locates the two-dimensional center of gravity at each peak location by integrating volume and moment sums within a user-defined radius and tolerance. The radius defines the integration area and the tolerance defines how accurately the center of gravity is to be determined. That is, the center of gravity calculation is iterated until the current value falls to within the prescribed tolerance of the previously calculated center of gravity value. Once the center of gravity positions on each side of the boundary are located, a calibration is performed by utilizing a linear least squares fit to the centers of gravity, inputting the interatomic distance, $s$, and then deriving the interplanar spacing, $d$. The width $W$ is determined by extrapolation to the planes closest to the boundary within the selected center of gravity peak arrays. Finally, the volume expansion $(\delta)$ is found by comparing the measured width $W$ to the effective unrelaxed width $W_{u}$ : where $\delta=W-W_{u}$. Since the lattice planes closest to the interface may be somewhat distorted, an option to delete specific planes and recalculate the least squares fit is used. For each measurement, subsequent peaks (starting at the first-selected peak closest to the boundary on each side) are deleted from the data set, and the least squares fit is recalculated. Figure 2 shows the resulting measurement of $\mathrm{W}$ versus number of planes deleted for a given measurement set. Note how $\mathrm{W}$ converges to a finite value as the number of planes deleted increases. Obviously, there is a maximum limit to how many planes may be deleted and still have a statistically adequate number of data points. We choose this limit to be the truncated value of $(\mathrm{N}-2) / 2$, where $\mathrm{N}$ is the number of peaks per side in the data set. The spread of values for $\mathrm{W}$ should also stay within a finite range for each data set under the following conditions: 1) an adequate number of planes (or peaks) have been used. (This number varies with the type of boundary and quality of image.) 2) there are no erratic data points. Erratic data points can easily spotted as anomalies in the $\mathrm{W}$ versus number of planes deleted graph.

\section{EVALUATION OF THE STATISTICAL ERROR}

The accuracy of the measurement technique was evaluated by using standard HREM multislice simulations of a Au $\sum 11$ (113) symmetric tilt boundary at various sample thickness and image defocus conditions. The rigid unrelaxed model was compared to a relaxed model using embedded atom potentials which included a volume expansion of $0.029 \mathrm{a}_{0}$. The two cases are illustrated in Figure 3: a) a rigid model of the Au $\Sigma 11$ (113) boundary with no volume expansion; and b) a relaxed model of the Au $\Sigma 11$ (113) boundary with a known volume expansion of $0.029 \mathrm{a}_{0}$. All images were analyzed under conditions which gave rise to 'white dots' at the atom positions. A small amount of shot noise $(\mathrm{S} / \mathrm{N}=12)$ was added to simulate typical HREM imaging conditions. Shown in Table II are the statistical results from a number of data sets (each set containing typically 50 measurements). The data illustrate that a standard error of better than $\pm 0.002 \mathrm{a}_{0}$ can be achieved under ideal conditions. 
The question remains: what are the 'ideal conditions'? Although the human error which existed previously in the technique has been minimized, other sources of error have been identified. One source arises from the distortion of the planes (peak positions) close to the boundary. In the relaxed boundary measurements, the volume expansion is not a constant rigid translation, but is composed of the summation of the displacements over a number of planes as one moves away from the boundary, as is expected in a relaxed boundary [16]. The displacement of individual planes as a function of distance from the grain boundary is often oscillatory in nature. Slight remnants of this behavior may be seen in the volume expansion measurement as an increasing number of planes are deleted from the data set (Figure 4). For this particular boundary, the measurement doesn't converge until at least five or six planes in the boundary region are excluded. Other boundaries which we have tested show similar behavior, although the number of planes which would need to be excluded varies somewhat.

In addition to variations of local displacements near the grain boundary, errors in the displacement measurement near the grain boundary core can arise from the HREM imaging conditions. This was found by image simulation of rigid models where, instead of local atomic relaxations, rigid shifts were applied to the planes on either side of the boundary. In this case, the planes near the boundary core also exhibited a distortion away from the known atomic column positions. This behavior was dependent on both defocus conditions and sample thickness, indicating the importance of selecting the proper imaging conditions. The data show that the more localized the imaging conditions [18], the more accurate the measurement. Therefore, a suitable defocus condition would be where the gradient of the aberration function ( $\operatorname{or} \operatorname{grad}(\chi)$ ) is equal to 0 , which is generally near Scherzer defocus as well. For the images analyzed in this paper, optimum defocus conditions typical for a JEM4000EX microscope operating at $400 \mathrm{kV}$ were slightly more negative than Scherzer defocus. It should also be noted that these types of errors could be minimized by ensuring that the measurement planes are far enough away from the boundary and that an adequate number of planes on both sides of the boundary are used in the data set.

Magnification of the digitized image was also a factor in the measurement accuracy. It was determined that a sampling of at least $0.05 \AA /$ pixel was required for the measurement accuracies we report here, which puts requirements on both the minimum magnification required in capturing the image (a magnification of approximately 700,000X or higher), and image processing capabilities to handle large image files (dimensions larger than 1024 pixels are generally required).

\section{EVALUATION OF THE IMAGE ACQUISITION ERROR}

There are a number of microscope aberrations which must be corrected for in order to properly interpret HREM images. Even small misalignments can drastically affect how the contrast is 
transferred. For example, both astigmatism and beam tilt can destroy the true symmetry in an image and may even add artificial periodicities [18]. Since astigmatism is easily identified, we will concentrate on two other critical alignments: beam tilt and crystal tilt.

The errors associated with image acquisition can be attributed mainly to the distortions on the image from the microscope misalignment. For example, Figure 5 illustrates what happens to an image as increasing amounts of beam tilt are added. Our data indicate that these distortions have a large effect on the center of gravity positions, thus introducing significant error into the evaluation of volume expansion.

As in the previous section, the effects of both beam and crystal tilt were investigated using the same simulated Au $\Sigma 11$ (113) boundary. Shown in Table III are the measured volume expansions for the boundary normalized to the lattice parameter $\left(a_{0}\right)$ under various imaging conditions. Crystal thicknesses of $5.0 \mathrm{~nm}$ and $8.3 \mathrm{~nm}$ were both considered. Beam tilt was introduced into the image in two independent directions (parallel and perpendicular to the boundary plane). Although beam tilt perpendicular to the boundary showed little effect up to $2 \mathrm{mrad}$ for a crystal thickness of $5.0 \mathrm{~nm}$, parallel to the boundary a negative deviation from ideal by nearly $79 \%$ is already seen with a beam tilt of only $1.0 \mathrm{mrad}$. These data illustrate the necessity of accurate beam tilt alignment for quantitative HREM analysis. Crystal tilt generally has less of a distorting effect on an image. In this case, up to 4 mrad parallel and perpendicular to the boundary showed generally less than a $10 \%$ change on the volume expansion measurement. Therefore, although crystal tilt is always undesirable, the technique is tolerant to the moderate amount of crystal misalignment which is sometimes unavoidable in HREM images of grain boundaries.

The intensity information in a typical HREM structure image can also be masked by both statistical and amorphous noise. In order to evaluate the effect of noise on the measurement accuracy, a varying amount of random noise was added to the simulated images of a relaxed boundary of known volume expansion of $0.029 \mathrm{a}_{0}$. The results are summarized in Table IV. The technique was found to be accurate to within $+/-0.002 a_{0}$ for signal-to-noise ratios $(S / N)$ greater than approximately 3.0 .

\section{CONCLUSION}

While only one particular type of boundary has been considered here, it serves to illustrate that the lattice fringe displacement method described in this paper allows the quantitative measurement of grain boundary volume expansion to better than $\pm 0.002 \mathrm{a}_{0}$. Proper imaging conditions and microscope alignment have been shown to be critical for accurate measurement. Images taken under localized imaging conditions yield the best results. Small deviations of the beam tilt alignments which may be 
barely noticeable in the image can give rise to significant errors in the measurement. The technique is less sensitive to small amounts of crystal tilt as well as shot noise in the image.

\section{ACKNOWLEDGMENTS}

The authors wish to thank D. Wolf, D. Ricker and K. Hogan for their assistance in this project. This work was supported by the U.S. Department of Energy, BES-Materials Sciences, under contract No. W-31-109-ENG-38.

\section{REFERENCES}

[1] M. J. Weins, H. Gleiter, and B. Chalmers, J. Appl. Phys. 42 (1971) 2639.

[2] D. Wolf, Acta Met. 37 (1989) 1983, 2823.

[3] D. Wolf, Acta Met. 38 (1990) 781, 791.

[4] R. C. Pond, Journ. of Microscopy 116 (1) (1979) 105.

[5] K Miyazawa and Y. Ishida, Joum. of Microscopy 142(2) (1986) 163.

[6] J. W. Matthews and W. M. Stobbs, Phil. Mag. 36(2) (1977) 373.

[7] C. Boothroyd, A. P. Crawley, and W. M. Stobbs, Phil. Mag. A 54(5) (1986) 663.

[8] J. N. Ness, W. M. Stobbs, and T. F. Page, Phil. Mag. A 54(5) (1986) 679.

[9] D. Rasmussen and C. B. Carter, Ultramicroscopy 32 (1990) 337.

[10] G. J. Wood, W. M. Stobbs, and D. J. Smith, Phil. Mag. A 50 (1984) 375.

[11] W. M. Stobbs, G. J. Wood, and D. J. Smith, Ultramicroscopy 14 (1985) 145.

[12] K. L. Merkle, Ultramicroscopy 40 (1992) 281.

[13] K. L. Merkle and D. Wolf, MRS Bulletin (Sept. 1990) 42.

[14] A. G. Crocker and B. A. Faridi, Acta Met. 28 (1980) 549.

[15] D. Wolf, private communication.

[16] S. P. Chen, D. J. Srolovitz, J. Materials Res. 4 (1989) 62.

[17] L. D. Marks, Ultramicroscopy 18 (1985) 33.

[18] D. J. Smith et al., Ultramicroscopy 11 (1983) 263. 
Figure Captions:

FIGURE 1. Schematic diagram of the lattice fringe displacement technique.

FIGURE 2. Measurement of the boundary width, $\mathrm{W}$, as a function of number of planes deleted from the data set. Known $\mathrm{W}=1.838 \mathrm{a}_{0}$.

FIGURE 3. a) rigid model of the Au $\Sigma 11$ (113) symmetric twin boundary, thickness $=5 \mathrm{~nm}$, defocus $=-70 \mathrm{~nm}$. b) relaxed model of the Au $\Sigma 11$ (113) symmetric twin boundary, thickness $=5 \mathrm{~nm}$, defocus $=-70 \mathrm{~nm}$. These are 'white dot' HREM images which were artifically contrast-enhanced to bring out the subtle differences between the two models.

FIGURE 4. Measurement of volume expansion as a function of how many planes are skipped in the boundary region. Note the oscillatory behavior in the volume expansion measurement as an increasing number of planes are deleted from the data set.

FIGURE 5. Image simulations of a Au $\sum 11$ (113) symmetric tilt boundary (thickness $=5 \mathrm{~nm}$, defocus $=-70 \mathrm{~nm}$ ) illustrating the distorting effect of increasing beam tilt; $x$ dimension is perpendicular to the boundary and $y$ dimension is parallel to the boundary. 
TABLE I. A Compilation of Experimental and Theoretical Results on the Measurement of Grain Boundary Volume Expansion in $\Sigma 3$ Twins.

$\begin{array}{llll}\text { Ref. Boundary Type } & \text { Measurement Technique } & \text { Measurement } / \text { Std. Error } \\ \text { [4] Al } \sum 3(121) \text { twin } & \alpha \text {-fringe displacement } & 0.02 \mathrm{~nm}(?) \\ {[6]} & \mathrm{Au} \sum 3(112) \text { twin } & \text { Moire fringe displacement } & 0.056 \mathrm{~nm}(?) \\ {[7] \quad \mathrm{Cu} \sum 3(111) \text { twin }} & \text { Fresnel fringe displacement } & 0.012 \mathrm{~nm}( \pm 0.002 \mathrm{~nm}) \\ {[10] \mathrm{Cu} \sum 3(111) \text { twin }} & \text { Lattice fringe displacement } & 0.001 \mathrm{~nm}( \pm 0.004 \mathrm{~nm}) \\ {[11] \mathrm{Au} \sum 3(111) \text { twin }} & \text { Lattice fringe displacement } & 0.009 \mathrm{~nm}( \pm 0.003 \mathrm{~nm}) \\ {[12] \mathrm{Au} \sum 3(111) \text { twin }} & \text { Lattice fringe displacement } & -0.0035 \mathrm{~nm}( \pm 0.004 \mathrm{~nm}) \\ {[14] \mathrm{Cu} \sum 3(111) \text { twin }} & \text { Simulation } & 0.0036 \mathrm{~nm} \\ {[15] \mathrm{Au} \sum 3(111) \text { twin }} & \text { Simulation } & -0.00016(\mathrm{EAM})\end{array}$

$\mathrm{EAM}=$ embedded atom potential $; \mathrm{LJ}=$ Lennard-Jones potential 
TABLE II. Volume Expansion Measurement Under Various Imaging Conditions.

All measurements made on a Au $\Sigma 11$ (113) symmetric tilt grain boundary of known $\delta=0.000$ for the rigid boundary and $\delta=0.029 \mathrm{a}_{0}$ for the relaxed boundary. All images exhibited 'white dot' contrast at the atom positions under the imaging conditions shown.

\begin{tabular}{cccc} 
Sample Thickness & Boundary Type & Image Defocus & Measured Volume Expansion \\
\hline$(\mathrm{nm})$ & & $(\mathrm{nm})$ & (normalized to $\left.\mathrm{a}_{0}\right)$ \\
& & & \\
5 & rigid & -60 & $0.001 \pm 0.002$ \\
5 & rigid & -70 & $0.002 \pm 0.002$ \\
8 & rigid & -70 & $0.000 \pm 0.002$ \\
& & & \\
5 & relaxed & -60 & $0.029 \pm 0.002$ \\
5 & relaxed & -70 & $0.030 \pm 0.001$ \\
5 & relaxed & -80 & $0.029 \pm 0.001$ \\
5 & relaxed & -10 & $0.032 \pm 0.003$ \\
5 & relaxed & -110 & $-0.264 \pm 0.007$ \\
8 & relaxed & -70 & $0.029 \pm 0.002$
\end{tabular}


TABLE III. Volume Expansion Measurement Under Various Imaging Conditions.

All measurements made on a Au $\Sigma 11$ (113) symmetric tilt grain boundary of known $\delta=0.029 a_{0}$ (relaxed boundary).

Sample Thickness Boundary Type Defocus Beam/Crystal Tilt Measured Volume Expansion

$(\mathrm{nm})$

$$
5
$$

5

5

5

5 relaxed

5 relaxed

8 relaxed

5

5

5 (nm)

$-70$

$-70$

$-70$

$-70$

$-70$

$-70$

$-70$

$-70$

$-70$

$-70$ (mrad)

none

1.6 (B perp.)

2.0 (B perp.)

3.3 (B perp.)

1.0 (B par.)

1.7 (B par.)

1.7 (B par.)

1.0 (C perp.)

4.0 (C perp.)

4.0 (C par.) (normalized to $\mathrm{a}_{0}$ )

$0.030 \pm 0.001$

$0.029 \pm 0.002$

$0.025 \pm 0.002$

$0.021 \pm 0.002$

$0.006 \pm 0.002$

$-0.001 \pm 0.002$

$-0.004 \pm 0.005$

$0.027 \pm 0.001$

$0.028 \pm 0.002$

$0.025 \pm 0.002$

$B=$ beam tilt; $C=$ crystal tilt; perp.= perpendicular to boundary; par. = parallel to boundary . 
TABLE IV. Measured Volume Expansion ( $\delta$ ) as a Function of Increasing Noise in the Image.

All measurements made on a $5 \mathrm{~nm}$ thick Au $\Sigma 11$ (113) symmetric tilt grain boundary of known $\delta=0.029 a_{0}$. Signal/Noise is defined as the average value of $S^{2}(x)$ divided by the average value of $\mathrm{N}^{2}(\mathrm{x})$, where $S$ and $N$ represent the signal and noise functions respectively.

Boundary Signal/Noise Measured Width (W) (normalized to $a_{0}$ )

relaxed

0 noise

14

12

4.8

3.0
$1.235 \pm 0.002$

$1.235 \pm 0.002$

$1.235 \pm 0.002$

$1.234 \pm 0.002$

$1.239 \pm 0.010$
Measured Vol. Exp. ( $(\delta)$

(normalized to $a_{0}$ )

$0.029 \pm 0.002$

$0.029 \pm 0.002$

$0.029 \pm 0.002$

$0.028 \pm 0.002$

$0.033 \pm 0.010$ 


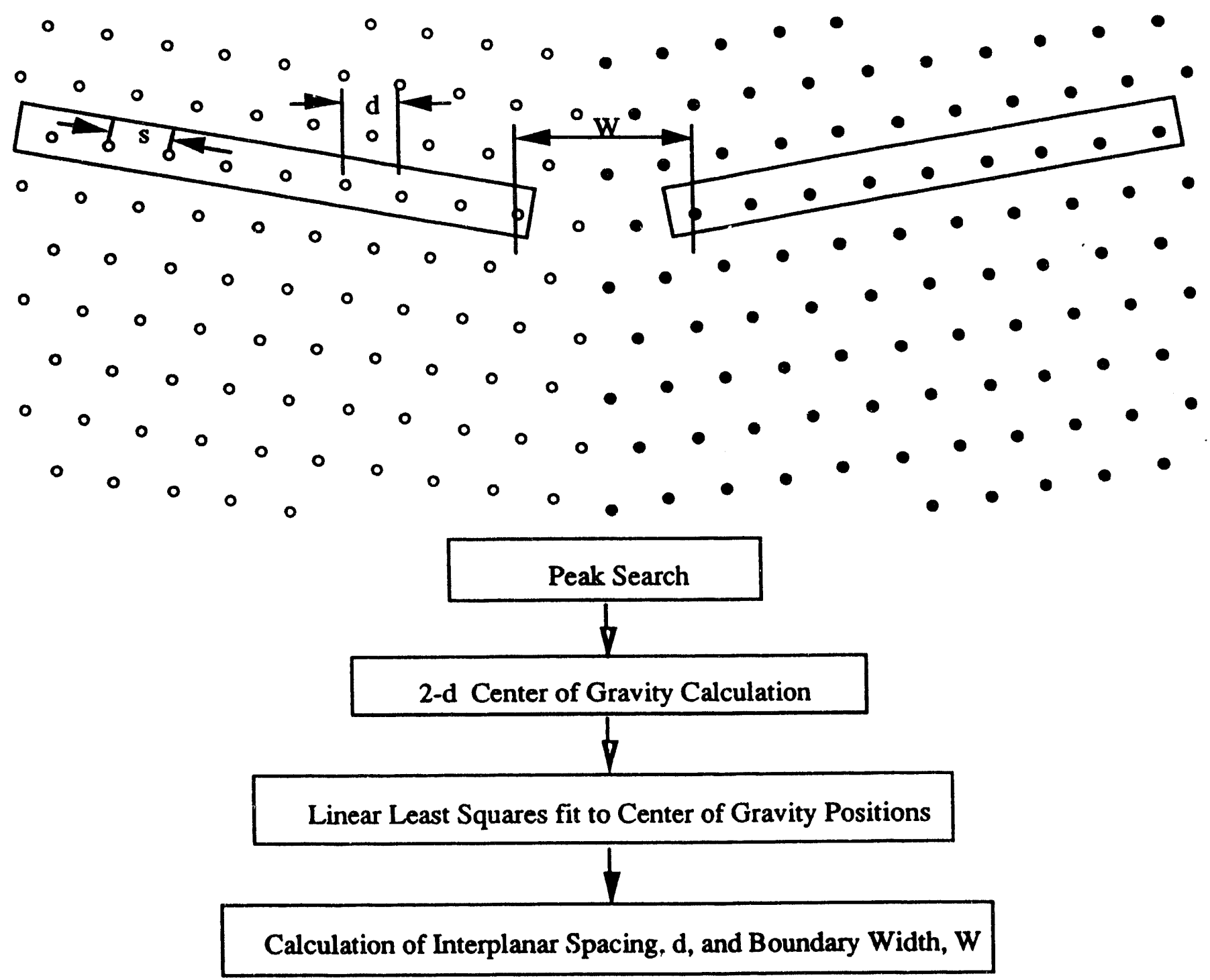




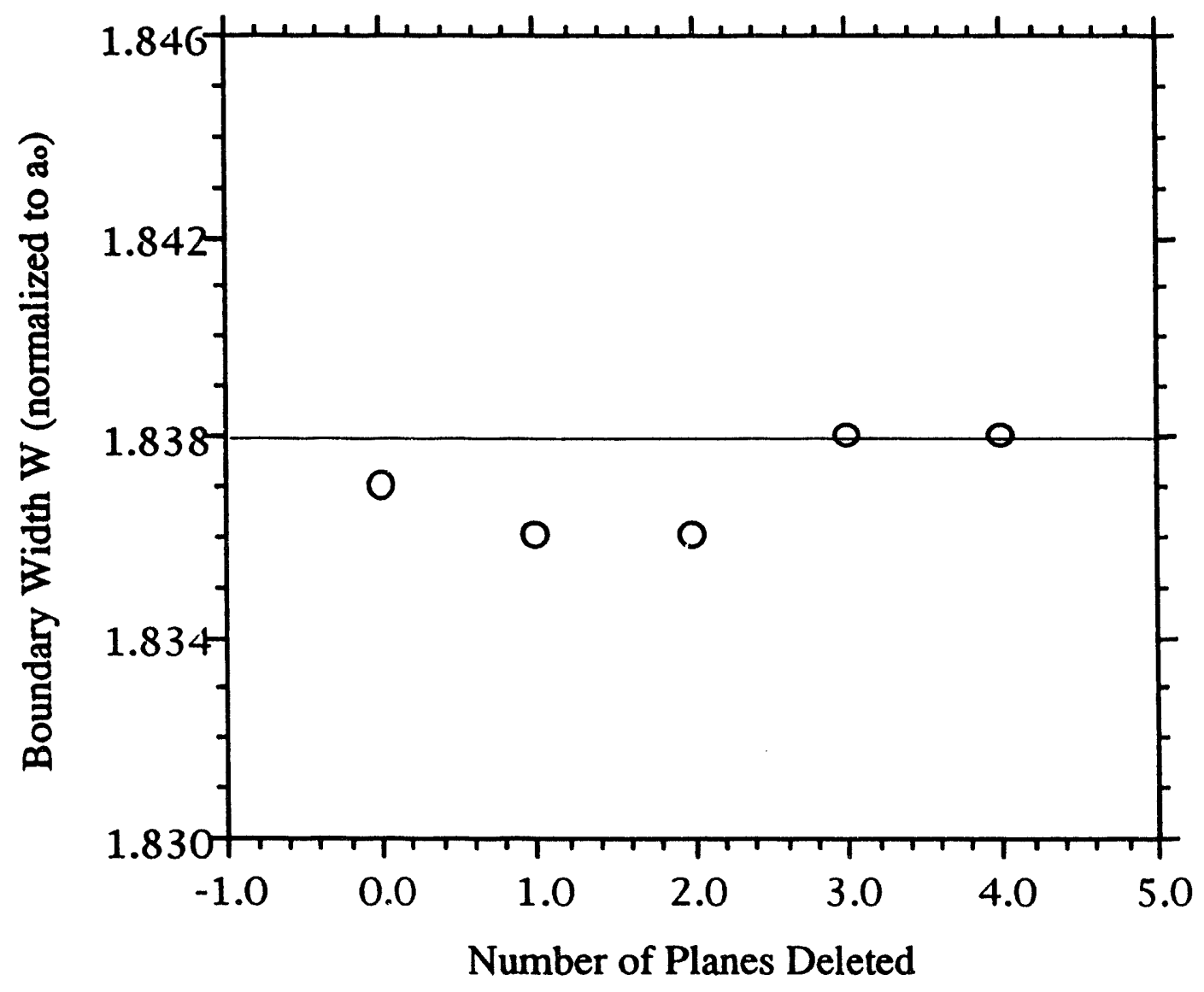




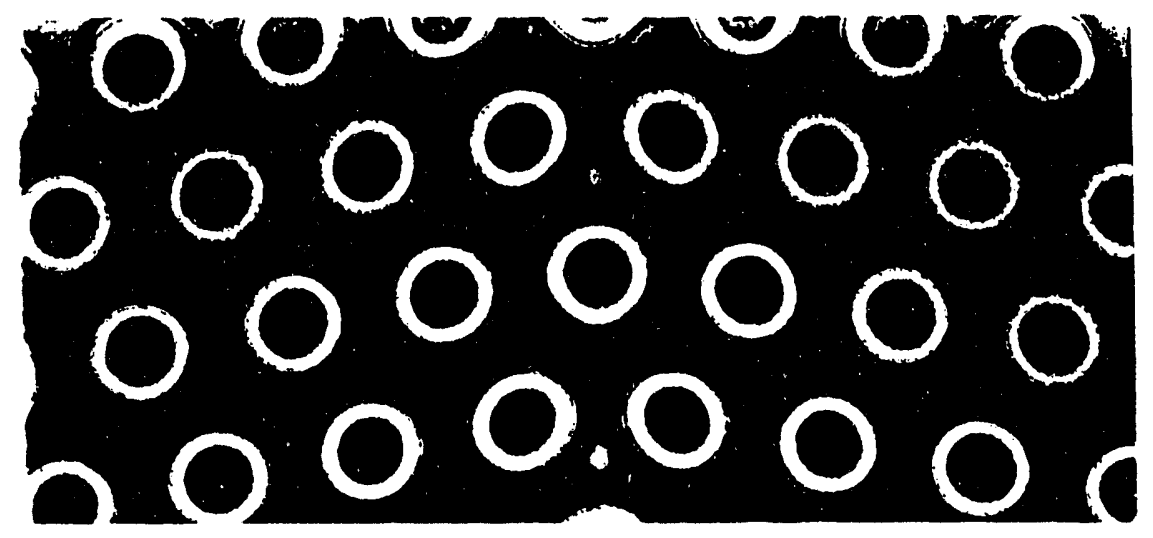

a) rigid model

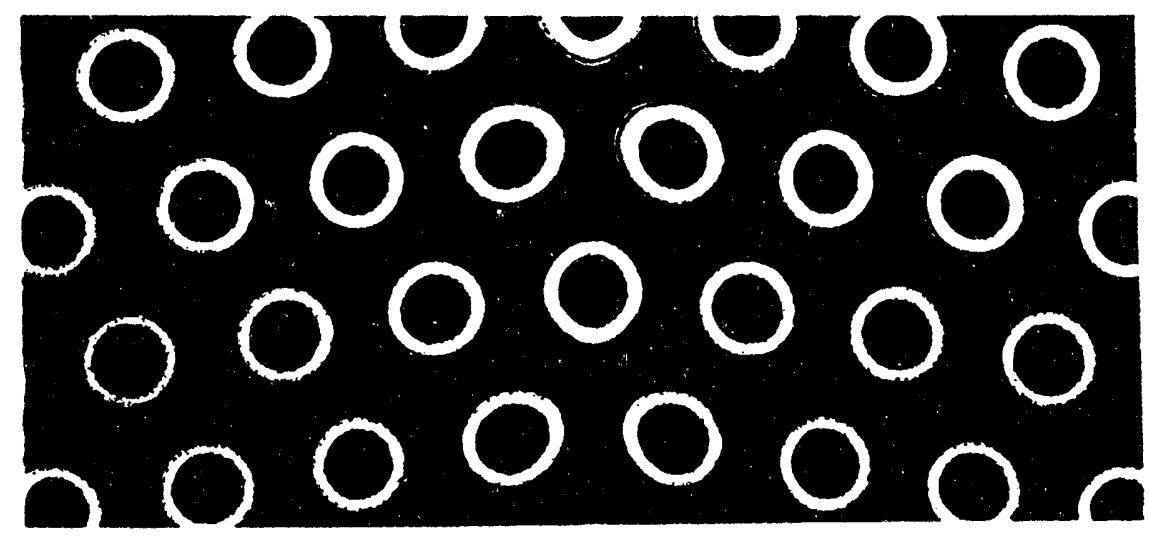

b) relaxed model 


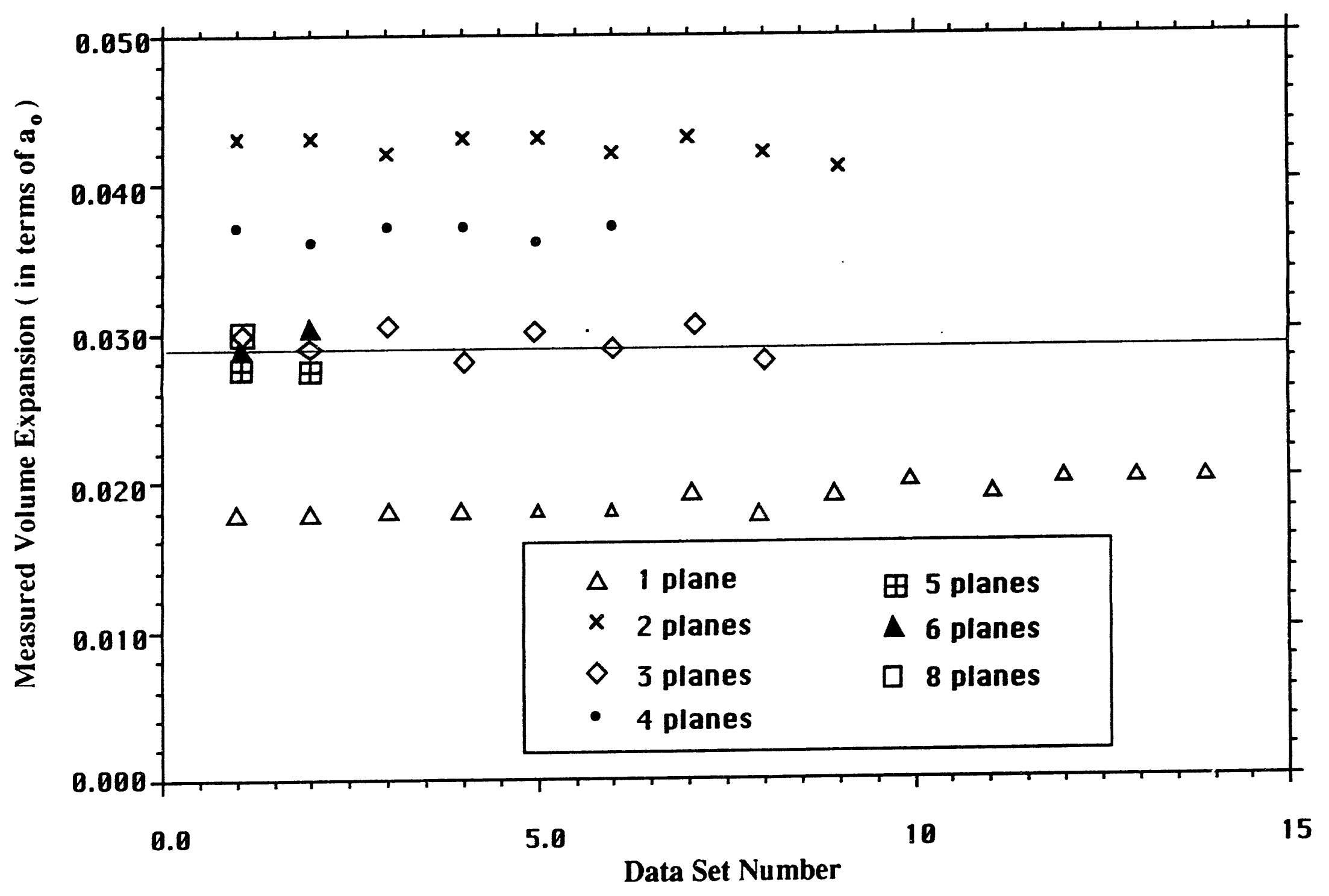


$\begin{array}{lllllllll}0 & 0 & 0 & 0 & 0 & 0 & 0 & 0 \\ 0 & 0 & 0 & 0 & 0 & 0 & 0 & 0\end{array}$ 200.000 - on a) no tilt

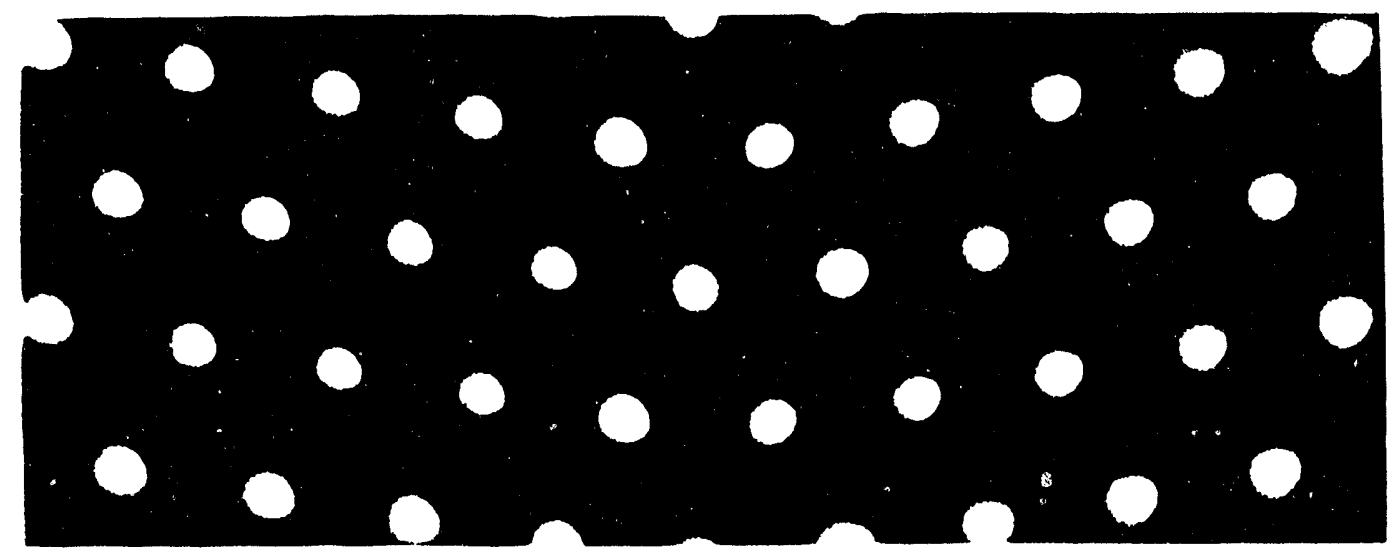
b) $2 \mathrm{mR}(\mathrm{x})$

- 000000 - 00000000 300000000 - o n 000 c) $3 \mathrm{mR}(\mathrm{x})$

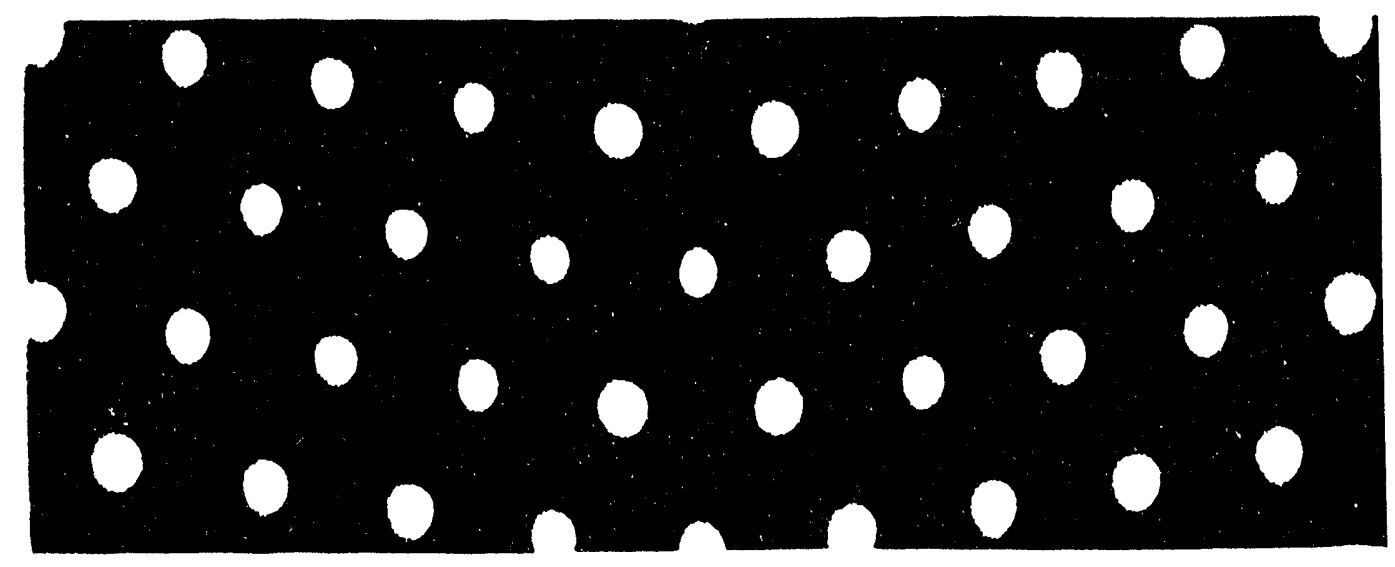

(d) $2 m R(y)$ 

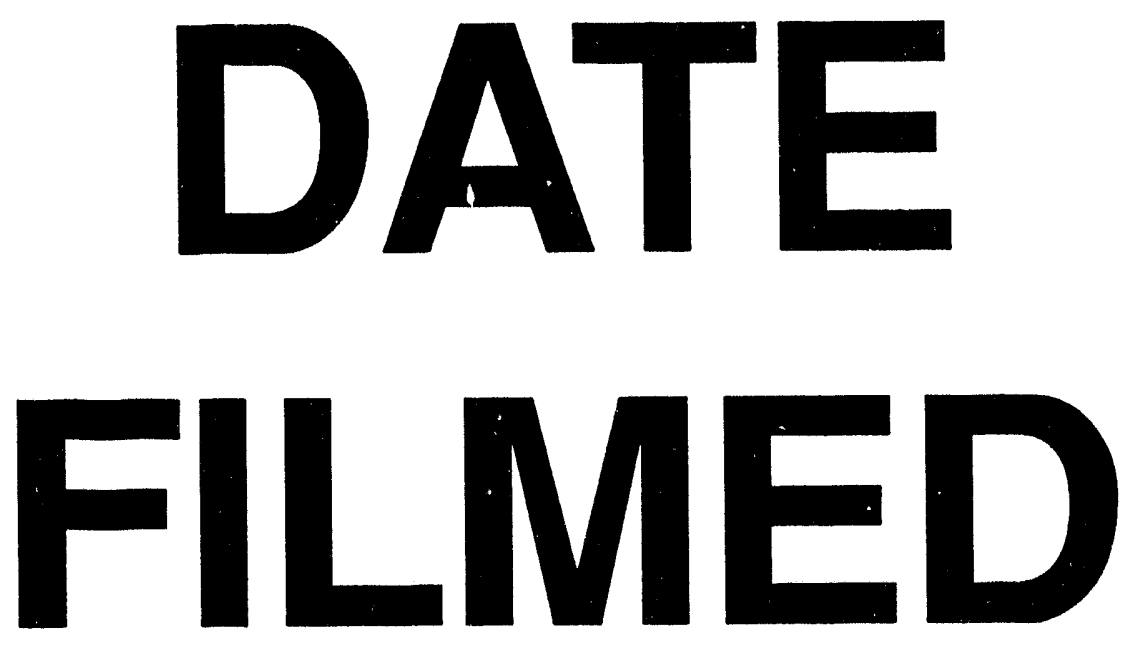

$6 / / / 94$
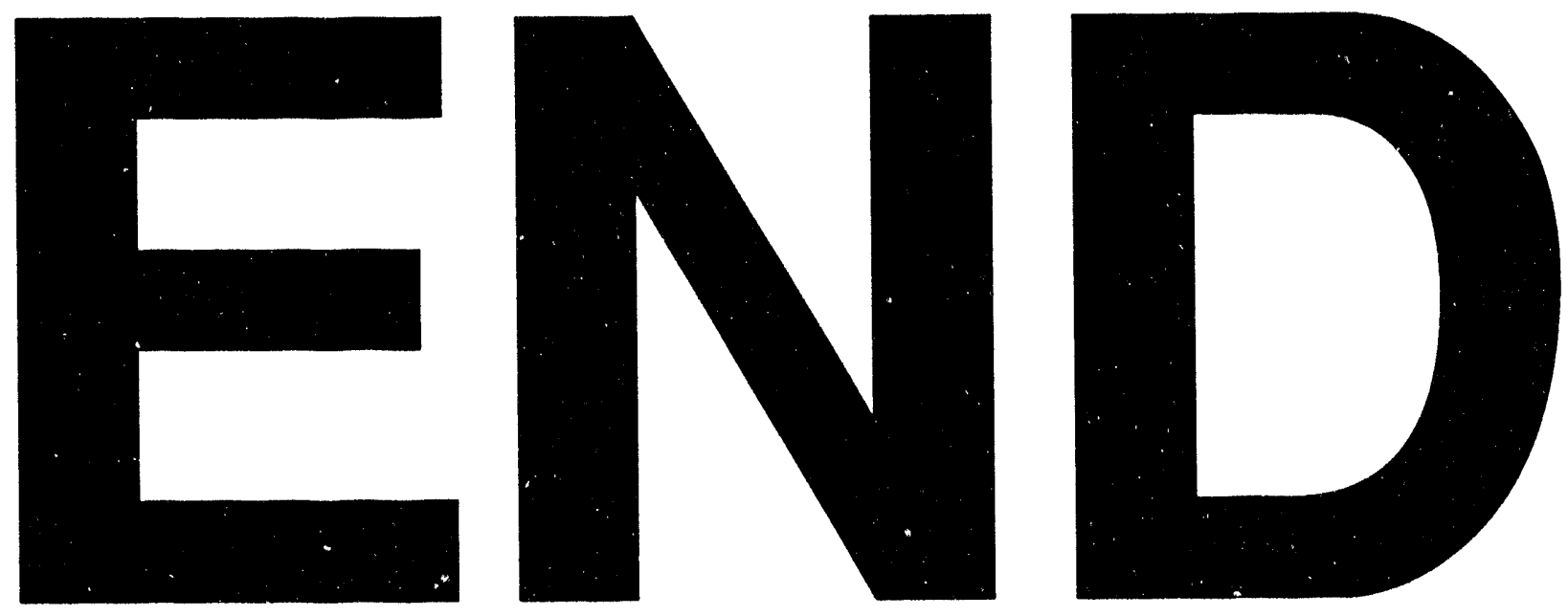


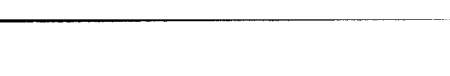

$\mid$

$\therefore$ 